\title{
Prevalence of Cardiac Disease in Breast Cancer Patients at Time of Diagnosis Compared to the General Female Population in Germany
}

\author{
Eva Lorenz $^{\mathrm{a}} \quad$ Maria Blettner $^{\mathrm{a}} \quad$ Björn Lange $^{\mathrm{b}} \quad$ Marcus Schmidt $^{\mathrm{c}} \quad$ Astrid Schneider $^{\mathrm{a}}$ \\ Lukas Schwentner $^{\mathrm{d}}$ Daniel Wollschläger $^{\mathrm{a}}$ Hiltrud Merzenich $^{\mathrm{a}}$ \\ a Institute of Medical Biostatistics, Epidemiology and Informatics, University Medical Center Mainz, Mainz, Germany; \\ ${ }^{b}$ Center for Cardiology, University Medical Center Mainz, Mainz, Germany; \\ ${ }^{\mathrm{c}}$ Department of Obstetrics and Gynecology, University Medical Center Mainz, Mainz, Germany; \\ ${ }^{\mathrm{d}}$ Department of Gynecology and Obstetrics, University Hospital UIm, Ulm, Germany
}

\section{Keywords}

Cardiovascular prevalence - Breast cancer patients .

German general population - Prevalence comparison .

Cardiovascular late effects

\section{Summary}

Background: Advances in oncological therapy have significantly improved breast cancer survival; therefore comorbid conditions are becoming more relevant. We investigated the prevalence of prior cardiovascular diseases and risk factors in patients with breast cancer compared to those in the general female population in Germany. Methods: The PASSOS heart study is a retrospective multicenter cohort study on cardiac late effects in breast cancer patients treated between 1998 and 2008. We analyzed the frequencies of cardiac diseases and cardiovascular risk factors in patients from this cohort as documented in anesthesia protocols compared to selfreported frequencies in the general female population in Germany. Results: 3,496 patients aged between 40 and 79 years who underwent breast surgery were considered for analysis. The age-standardized prevalence of cardiac diseases or cardiovascular risk factors was 6.75 versus $7.52 \%$ and 69 versus $80.92 \%$, respectively. Coronary heart disease (3.96 vs. $5.18 \%$ ) and angina pectoris $(0.37$ vs. $1.03 \%$ ) prevalence was lower in breast cancer patients, while non-fatal myocardial infarction (2.06 vs. $1.81 \%$ ) and stroke (2.64 vs. $2.34 \%$ ) were more frequent (not statistically significant). Conclusion: Pre-existing cardiac diseases and cardiovascular risk factors are common in both study populations, being slightly less fre- quent in the PASSOS cohort. When making therapy decisions, the cardiac risk profile should be carefully monitored and taken into account.

(c) 2018 S. Karger GmbH, Freiburg

\section{Introduction}

Breast cancer is the most frequent cancer among women in Germany, with approximately 72,000 annual incident cases. Despite an increasing number of incident cases, breast cancer mortality in Germany has significantly decreased in the past 10 years due to advances in oncological therapy $[1,2]$. Cancer survivors are on average 64 years old at the time of diagnosis, and their ageing process is frequently accompanied by comorbid conditions [1]. The prevalence of prior cardiac disease and of cardiovascular risk factors such as coronary heart disease, myocardial infarction, diabetes mellitus, arterial hypertension, hyperlipoproteinemia, and obesity rises with increasing age at cancer diagnosis [3].

In addition to the age-related cardiovascular risks, cardiotoxic late effects induced by chemotherapeutic treatment with anthracyclines and by radiation therapy must be considered $[4,5]$. The presence of pre-existing cardiac diseases and risk factors adversely affects breast cancer survival, independent of specific therapy-related cardiotoxic effects [6]. A detailed anamnesis and an assessment of individual cardiovascular risk profiles are therefore highly important when monitoring breast cancer patients $[4,5]$.

A descriptive overview of the cardiac disease and risk factor prevalence in a large breast cancer study conducted in Hamburg

\section{KARGER}

(c) 2018 S. Karger GmbH, Freiburg 
Table 1. Demographic characteristics, cardiac diseases, and cardiovascular risk factors considered for prevalence comparisons based on data from the PASSOS heart study and DEGS1 (German Health Interview and Examination Survey for Adults)

\begin{tabular}{|c|c|c|}
\hline Covariate & PASSOS heart study (1998-2008) & DEGS1 (2008-2011) \\
\hline \multicolumn{3}{|l|}{ Cardiac disease } \\
\hline Coronary heart disease & anaesthesia protocol (yes/no) & self-reported (yes/no) \\
\hline Myocardial infarction & anaesthesia protocol (yes/no) & self-reported (yes/no) \\
\hline Angina pectoris & anaesthesia protocol (yes/no) & $\begin{array}{l}\text { self-reported (yes/no), only documented if coronary heart disease was } \\
\text { answered with 'yes' }\end{array}$ \\
\hline Stroke & anaesthesia protocol (yes/no) & self-reported (yes/no) \\
\hline \multicolumn{3}{|l|}{ Cardiovascular risk factor } \\
\hline Arterial hypertension & $\begin{array}{l}\text { systolic blood pressure } \geq 140 \mathrm{~mm} \mathrm{Hg} \\
\text { diastolic blood pressure } \geq 90 \mathrm{~mm} \mathrm{Hg} \\
\text { and/or antihypertensive medication }\end{array}$ & $\begin{array}{l}\text { systolic blood pressure } \geq 140 \mathrm{~mm} \mathrm{Hg} \text {, diastolic blood pressure } \geq 90 \mathrm{~mm} \mathrm{Hg} \\
\text { (mean from second and third DEGS1 measurement) and/or antihypertensive } \\
\text { medication }\end{array}$ \\
\hline Diabetes mellitus & anaesthesia protocol (yes/no) & self-reported (yes/no) \\
\hline Hyperlipoproteinemia & anaesthesia protocol (yes/no) & self-reported (yes/no) \\
\hline $\begin{array}{l}\text { Overweight or obesity } \\
\text { (body mass index } \geq 25 \text { ) }\end{array}$ & $\begin{array}{l}\text { anaesthesia protocol } \\
\text { (self-reported) }\end{array}$ & self-reported (yes/no) \\
\hline \multicolumn{3}{|l|}{ Baseline characteristic } \\
\hline Age, years & at time of breast cancer diagnosis & at time of survey \\
\hline
\end{tabular}

and the Rhine-Neckar region was published recently [7]. The authors investigated determinants of incident cardiovascular diseases in breast cancer survivors and reported implications regarding prevention strategies. The authors observed an increased risk for cardiovascular diseases which was positively associated with age, body mass index, and intake of aromatase inhibitors (hazard ratio (HR) 1.42, confidence interval (CI) 1.09-1.84).

In the following analysis, pre-existing cardiac diseases and risk factors were evaluated in 3,496 breast cancer patients from a retrospective epidemiological cohort study $[8,9]$. We examined the prevalence of cardiac diseases and cardiovascular risk factors at the time of breast cancer diagnosis, i.e. before the onset of oncological therapy. Furthermore, we compared the prevalence of pre-existing cardiac diseases and risk factors in breast cancer patients with the general female population in Germany. Such a comparison has not been reported before.

\section{Patients and Methods}

\section{Study Population: PASSOS Heart Study}

A total of 11,982 breast cancer patients were recruited at the University Medical Centers Mainz and Ulm as well as 16 associated Ulm network clinics $[10,11]$. Women were eligible for participation in the study if they were diagnosed with primary breast cancer between 1998 and 2008 with no metastases at the time of diagnosis and had undergone breast surgery. The cohort comprised information on all clinically relevant therapies. In a mortality follow-up, the vital status as well as the recorded cause of death were determined up until December 31, 2012 [8].

In addition to treatment and diagnostic data, information from anesthesia protocols were recorded in the 2 study centers Ulm and Mainz if breast surgery was performed. The anesthesia protocols comprised basic data on preoperative conditions, premedication, information on cardiac comorbidities, and cardiovascular risk factors at the time of breast cancer diagnosis. The information was recorded by the treating physicians.

\section{Study Population: DEGS1}

For a prevalence comparison between the PASSOS heart study and the general female German population, data from the 'German Health Interview and Examination Survey for Adults' (DEGS) were used. The study is part of the health monitoring by the Robert Koch Institute (RKI). The first wave of the survey (DEGS1) was conducted between 2008 and 2011 and included both interviews and medical examinations. The target population comprised participants living in Germany aged between 18 and 79 years. Interviews and an additional medical examination were performed on 7,238 participants [12]. Information on cardiovascular diseases was collected only in subjects over 40 years of age ( $\mathrm{n}=5,901)$, which included 3,110 women who were eligible for our comparison with patients from the PASSOS heart study [13].

In order to ensure comparable age ranges, 3,496 breast cancer patients between 40 and 79 years of age at the time of diagnosis who had undergone breast surgery were eligible for a prevalence comparison. Variables considered for the prevalence comparison are given in table 1 .

\section{Statistical Methods}

The distribution of age, year of diagnosis, and tumor stage are presented for both the Mainz and the Ulm study center. Relative frequencies for dichotomous outcomes (myocardial infarction, coronary heart disease, angina pectoris, stroke, arterial hypertension, hyperlipoproteinemia, diabetes mellitus, and overweight or obesity) were reported for women aged 40-49, 50-59, 60-69, and 70-79 years. In addition, crude and age-standardized prevalences were calculated. Prevalences were calculated as percentages of participants who had been identified as having the outcome in relation to the total number of all participants (answered 'yes' compared to 'no', 'unknown', or missing data) with 95\% CI. Age standardization was performed based on the general female German population in 2011 (census 2011, Federal Statistical Office, call date: 14/07/2016, data license by-2-0).

For categorical variables, number and percentages of patients are presented. Percentages are based on all observations including missing values. In DEGS1, angina pectoris was only evaluated if coronary heart disease was present. Similarly, the prevalence of angina pectoris in breast cancer patients only includes patients with coronary heart disease. Missing values for angina pectoris are only reported in patients with coronary heart disease, not in patients without coronary heart disease as these patients were not interviewed with regard to the occurrence of angina pectoris in DEGS1.

Differences in the presence of possible risk factors and pre-existing cardiovascular diseases between breast cancer patients and the female general popula- 
Table 2. Distribution of age, year of diagnosis, tumor stage, and nodal stage in 3,496 patients from the PASSOS heart study in Mainz and Ulm

\begin{tabular}{lc}
\hline & Total, $\mathrm{n}(\%)$ \\
\hline Age & $785(22.5)$ \\
$40-49$ & $1,008(28.8)$ \\
$50-59$ & $1,105(31.6)$ \\
$60-69$ & $597(17.1)$ \\
$70-79$ & $1(0)$ \\
Missing values & \\
\hline Year of diagnosis & $470(13.4)$ \\
$1998-2000$ & $859(24.6)$ \\
$2001-2003$ & $1,080(30.9)$ \\
$2004-2006$ & $1,087(31.1)$ \\
$2007-2008$ & \\
\hline
\end{tabular}

T stage

0

$8(0.2)$

$1,718(49.1)$

$1,056(30.2)$

$167(4.8)$

$164(4.7)$

$298(8.5)$

In situ

$85(2.4)$

\begin{tabular}{lc}
\hline N stage & \\
0 & $2,027(58)$ \\
1 & $900(25.7)$ \\
2 & $192(5.5)$ \\
3 & $123(3.5)$ \\
Missing values & $254(7.3)$ \\
\hline Sum & $3,496(100)$ \\
\hline
\end{tabular}

tion in Germany were examined by comparing $\mathrm{CI}$ of prevalence (test for overlapping $\mathrm{CI}$ ).

Multivariate Cox regression was used to investigate the association between prior cardiovascular risk factors and the overall mortality risk. HRs and corresponding 95\% CI were estimated. The analysis was adjusted for the potential confounders age at diagnosis and tumor laterality and stratified for a prior diagnosis of cardiac illness.

A comparison between breast cancer patients, deceased before 2013, and the general German population was conducted as a sensitivity analysis.

\section{Results}

A total of 3,496 breast cancer patients aged between 40 and 79 years who had undergone breast surgery were considered for the prevalence comparison. Median age was 58.9 years at breast cancer diagnosis. The majority of patients in the Mainz and Ulm study centers were diagnosed after the year 2000 (table 2). In accordance with the inclusion criteria which ensured recruitment of patients without metastases, a favorable stage of breast cancer was observed in the PASSOS cohort. Tumor stage T1 was reported in $52.8 \%$ of the patients from Mainz and in $44.5 \%$ of the patients from Ulm. Node-negative status was observed in 60.1 and $54.7 \%$ of patients in Mainz and Ulm, respectively (table 2).

After age standardization, the overall prevalence of coronary heart disease was $3.96 \%$ in PASSOS patients, compared to $5.18 \%$ in the general female population. The age-standardized prevalence of myocardial infarction was 2.06 versus $1.81 \%$, that of angina pectoris was 0.37 versus $1.03 \%$, and that of stroke was 2.64 versus $2.34 \%$. $6.75 \%$ of the PASSOS patients versus $7.25 \%$ of the general female population had at least one cardiac disease prior to the oncological treatment (table 3).

Overall, cardiac disease prevalence increased with increasing age, both in PASSOS patients and in the general female population (fig. 1, table 3). However, a comparison of both study populations within individual age groups showed diverging patterns. In women aged 60-69 years, a lower prevalence of coronary heart disease and myocardial infarction was observed in PASSOS patients compared to the control population (coronary heart disease: 4.16 vs. $8.45 \%$, heart attack: 1.99 vs. $2.9 \%$ ). The prevalence of non-fatal myocardial infarction was higher in breast cancer patients aged 70-79 years compared to the DEGS1 population (heart attack: 7.04 vs. $4.71 \%$ ). Only few cases were observed in younger women in both study populations; therefore, no conclusions can be drawn for coronary heart disease and myocardial infarction (table 3).

The incidence of angina pectoris was consistently lower among breast cancer patients across all age groups (fig. 1). The incidence of stroke was consistently higher among breast cancer patients for women aged between 40 and 69 years (fig. 1).

Cardiovascular risk factors were overall lower or in some way comparable across all age groups. After age standardization, the prevalence of arterial hypertension was $35.15 \%$ in breast cancer patients and $38.49 \%$ in the general female population. For hyperlipoproteinemia, the respective values were 28.28 versus $34.84 \%$. The age-standardized prevalence of diabetes mellitus was 7.55 versus $8.94 \%$, the prevalence of overweight or obesity was 51.77 versus $66.92 \%$. The age-standardized prevalence of the presence of at least one of the cardiovascular disease risk factors was 69 and $80.92 \%$, respectively (table 4).

The prevalence of cardiovascular risk factors (arterial hypertension, hyperlipoproteinemia, diabetes mellitus, and overweight or obesity) rose with increasing age in both study populations (fig. 2, table 4). Lower prevalences were observed for arterial hypertension in breast cancer patients across all age groups, while the hyperlipoproteinemia prevalence was lower in women aged over 50 years, with more pronounced differences in the age groups 60-69 and 70-79 years (60-69: 35.29 vs. $48.17 \%$, 70-79: 38.02 vs. $53.22 \%$ ). Diabetes mellitus prevalence was lower in breast cancer patients between the ages of 40 and 49 years (1.53 vs. $5.1 \%$ ), while no differences were found in women older than 50 years. 'Overweight or obesity' were observed less often in breast cancer patients across all age groups. The proportion of women who were overweight or obese increased with increasing age.

\section{Discussion}

For our prevalence comparison, we considered 3,496 breast cancer patients from the PASSOS heart study between the ages of 40 and 79 who were recruited at the hospitals in Mainz and Ulm. The prevalence of pre-existing cardiac diseases and risk factors de- 
Table 3. Prevalence of prior cardiac diseases stratified by age; crude and age-standardized prevalence (PASSOS heart study vs. DEGS1)

\begin{tabular}{|c|c|c|c|c|c|c|c|c|c|}
\hline \multirow[t]{2}{*}{ Age, years } & \multirow[t]{2}{*}{ Cardiac disease } & \multicolumn{4}{|c|}{ PASSOS } & \multicolumn{4}{|c|}{ DEGS1 (CAPI) } \\
\hline & & $\mathrm{N}$ & $\mathrm{n}$ & $\%$ & $95 \% \mathrm{CI}$ & $\mathrm{N}$ & $\mathrm{n}$ & $\%$ & $95 \% \mathrm{CI}$ \\
\hline \multirow[t]{5}{*}{$40-49$} & coronary heart disease & 785 & 1 & 0.13 & $-0.12 ; 0.38$ & 823 & 8 & $0.97^{\mathrm{b}}$ & $0.30 ; 1.64$ \\
\hline & myocardial infarction & & 1 & 0.13 & $-0.12 ; 0.38$ & & 3 & $0.36^{\mathrm{c}}$ & $-0.05 ; 0.78$ \\
\hline & angina pectoris & & 0 & 0.00 & $0.00 ; 0.00$ & & 3 & $0.36^{\mathrm{d}}$ & $-0.05 ; 0.78$ \\
\hline & stroke & & 6 & 0.76 & $0.16 ; 1.37$ & & 6 & $0.73^{e}$ & $0.15 ; 1.31$ \\
\hline & any cardiac disease & & 8 & 1.02 & $0.32 ; 1.72$ & & 14 & 1.70 & $0.82 ; 2.58$ \\
\hline \multirow[t]{5}{*}{$50-59$} & coronary heart disease & 1,008 & 17 & 1.69 & $0.89 ; 2.48$ & 857 & 14 & $1.63^{b}$ & $0.78 ; 2.48$ \\
\hline & myocardial infarction & & 5 & 0.50 & $0.06 ; 0.93$ & & 1 & $0.12^{\mathrm{c}}$ & $-0.11 ; 0.35$ \\
\hline & angina pectoris & & 2 & 0.20 & $-0.08 ; 0.47$ & & 3 & $0.35^{\mathrm{d}}$ & $-0.05 ; 0.75$ \\
\hline & stroke & & 13 & 1.29 & $0.59 ; 1.99$ & & 5 & $0.58^{\mathrm{e}}$ & $0.07 ; 1.09$ \\
\hline & any cardiac disease & & 30 & 2.98 & $1.93 ; 4.03$ & & 19 & 2.22 & $1.23 ; 3.20$ \\
\hline \multirow[t]{5}{*}{$60-69$} & coronary heart disease & 1,105 & 46 & 4.16 & $2.99 ; 5.34$ & 793 & 67 & $8.45^{b}$ & $6.51 ; 10.38$ \\
\hline & myocardial infarction & & 22 & 1.99 & $1.17 ; 2.81$ & & 23 & $2.90^{c}$ & $1.73 ; 4.07$ \\
\hline & angina pectoris & & 5 & 0.45 & $0.06 ; 0.85$ & & 12 & $1.51^{\mathrm{d}}$ & $0.66 ; 2.36$ \\
\hline & stroke & & 42 & 3.80 & $2.67 ; 4.93$ & & 23 & $2.90^{\mathrm{e}}$ & $1.73 ; 4.07$ \\
\hline & any cardiac disease & & 94 & 8.51 & $6.86 ; 10.15$ & & 93 & 11.73 & $9.49 ; 13.97$ \\
\hline \multirow[t]{5}{*}{$70-79$} & coronary heart disease & 597 & 75 & 12.56 & $9.9 ; 15.22$ & 637 & 78 & $12.24^{\mathrm{b}}$ & $9.70 ; 14.79$ \\
\hline & myocardial infarction & & 42 & 7.04 & $4.98 ; 9.09$ & & 30 & $4.71^{c}$ & $3.06 ; 6.35$ \\
\hline & angina pectoris & & 6 & 1.01 & $0.20 ; 1.81$ & & 14 & $2.20^{\mathrm{d}}$ & $1.06 ; 3.34$ \\
\hline & stroke & & 37 & 6.20 & $4.26 ; 8.13$ & & 40 & $6.28^{\mathrm{e}}$ & $4.40 ; 8.16$ \\
\hline & any cardiac disease & & 111 & 18.59 & $15.47 ; 21.71$ & & 116 & 18.21 & $15.21 ; 21.21$ \\
\hline \multirow{5}{*}{$\begin{array}{l}\text { Total, } \\
\text { crude }\end{array}$} & coronary heart disease & 3,496 & 139 & 3.98 & $3.33 ; 4.62$ & 3,110 & 167 & 5.37 & $4.58 ; 6.16$ \\
\hline & myocardial infarction & & 70 & 2.00 & $1.54 ; 2.47$ & & 57 & 1.83 & $1.36 ; 2.30$ \\
\hline & angina pectoris & & 13 & 0.37 & $0.17 ; 0.57$ & & 32 & 1.03 & $0.67 ; 1.38$ \\
\hline & stroke & & 98 & 2.80 & $2.26 ; 3.35$ & & 74 & 2.38 & $1.84 ; 2.92$ \\
\hline & any cardiac disease & & 243 & 6.95 & $6.11 ; 7.79$ & & 242 & 7.78 & $6.84 ; 8.72$ \\
\hline \multirow{5}{*}{$\begin{array}{l}\text { Total, } \\
\text { age- } \\
\text { adjusted }\end{array}$} & coronary heart disease & 3,496 & 138 & 3.96 & $3.31 ; 4.61$ & 3,110 & 161 & 5.18 & $4.42 ; 5.94$ \\
\hline & myocardial infarction & & 72 & 2.06 & $1.57 ; 2.54$ & & 56 & 1.81 & $1.34 ; 2.28$ \\
\hline & angina pectoris & & 13 & 0.37 & $0.16 ; 0.58$ & & 32 & 1.03 & $0.67 ; 1.40$ \\
\hline & stroke & & 92 & 2.64 & $2.11 ; 3.18$ & & 73 & 2.34 & $1.81 ; 2.87$ \\
\hline & any cardiac disease & & 236 & 6.75 & $5.94 ; 7.57$ & & 234 & 7.52 & $6.62 ; 8.41$ \\
\hline Sum & & $3,496^{\mathrm{a}}$ & & & & 3,110 & & & \\
\hline
\end{tabular}

a'Missing age information for 1 patient from PASSOS heart study.

${ }^{b}$ Missing values for coronary heart disease in DEGS in 0.85\% (40-49), 1.86\% (50-59), 2.78\% (60-69), 3.92\% (70-79).

${ }^{\mathrm{c}}$ Missing values for myocardial infarction in DEGS in 0.73\% (40-49), 0.82\% (50-59), 1.39\% (60-69), 2.04\% (70-79).

${ }^{\mathrm{d}}$ Missing values for angina pectoris in DEGS in 0\% (40-49), 0.12\% (50-59), 0.25\% (60-69), 0.63\% (70-79).

${ }^{\mathrm{e}}$ Missing values for stroke in DEGS in 0.73\% (40-49), 1.05\% (50-59), 1.14\% (60-69), 2.04\% (70-79).

fInformation on angina pectoris DEGS was assessed only if coronary heart disease was diagnosed. Prevalences reported in PASSOS were derived accordingly.

Numbers in italics: Non-overlapping CIs.

DEGS1 = German Health Interview and Examination Survey for Adults; CAPI = computer-assisted personal interview; $\mathrm{N}=$ population size in age groups; $\mathrm{n}=$ outcome frequency in age groups; $\mathrm{CI}=$ confidence interval.

termined at the time of breast cancer diagnosis were compared to those in a subset of the DEGS1 study population representative of the general female population.

Lower prevalences of coronary heart disease and angina pectoris were observed in breast cancer patients. Overall, only slight differences were observed for the prevalence of myocardial infarction and stroke between the 2 study populations (table 2 ).

The risk factors arterial hypertension, hyperlipoproteinemia, diabetes mellitus, and overweight or obesity were observed less often in breast cancer patients (table 4). Differences were more pronounced for arterial hypertension and hyperlipoproteinemia than for diabetes mellitus.

Merzenich et al. [8] showed that a prior diagnosis of cardiac illness increased the overall mortality risk. A modified multivariate analysis to investigate the association between prior cardiovascular risk factors and the overall mortality risk showed that the presence of any cardiovascular risk factor was positively associated with an increased overall mortality risk (HR 1.21, 95\% CI 1.06-1.37).

In summary, breast cancer patients in our cohort had a more favorable health status with regard to cardiac diseases and cardio- 
Fig. 1. Prevalence of cardiac diseases at the time of breast cancer diagnosis, stratified by age (PASSOS vs. DEGS1).
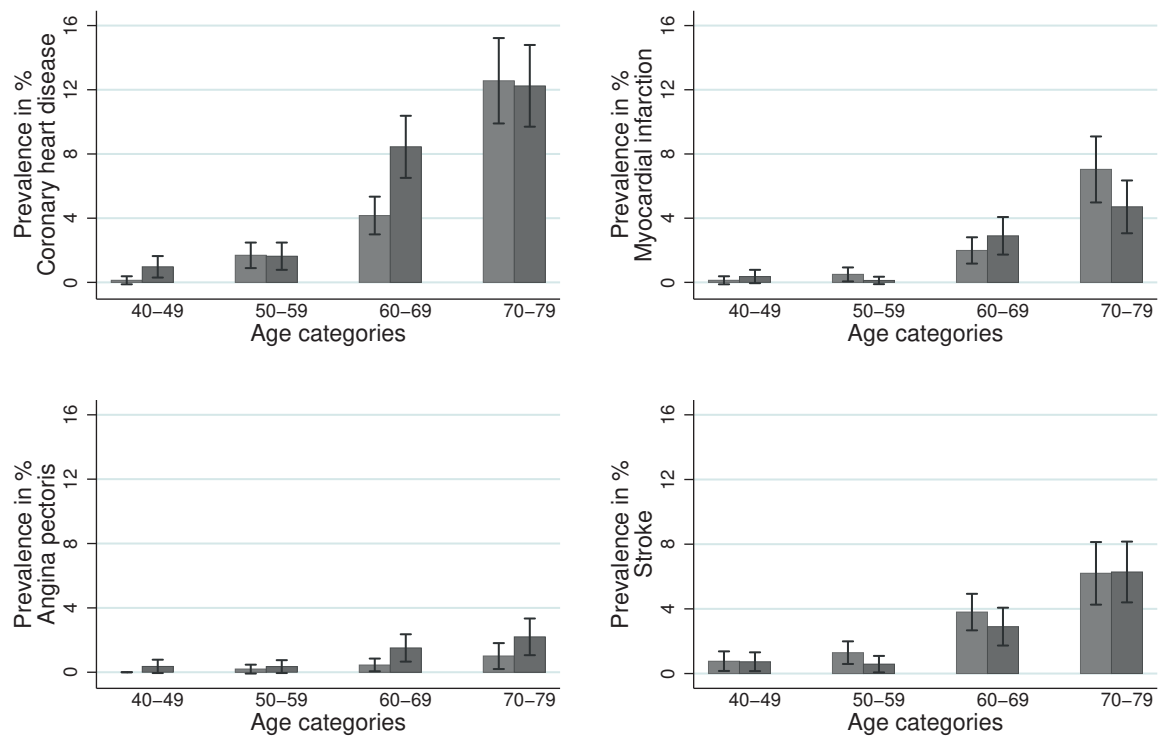
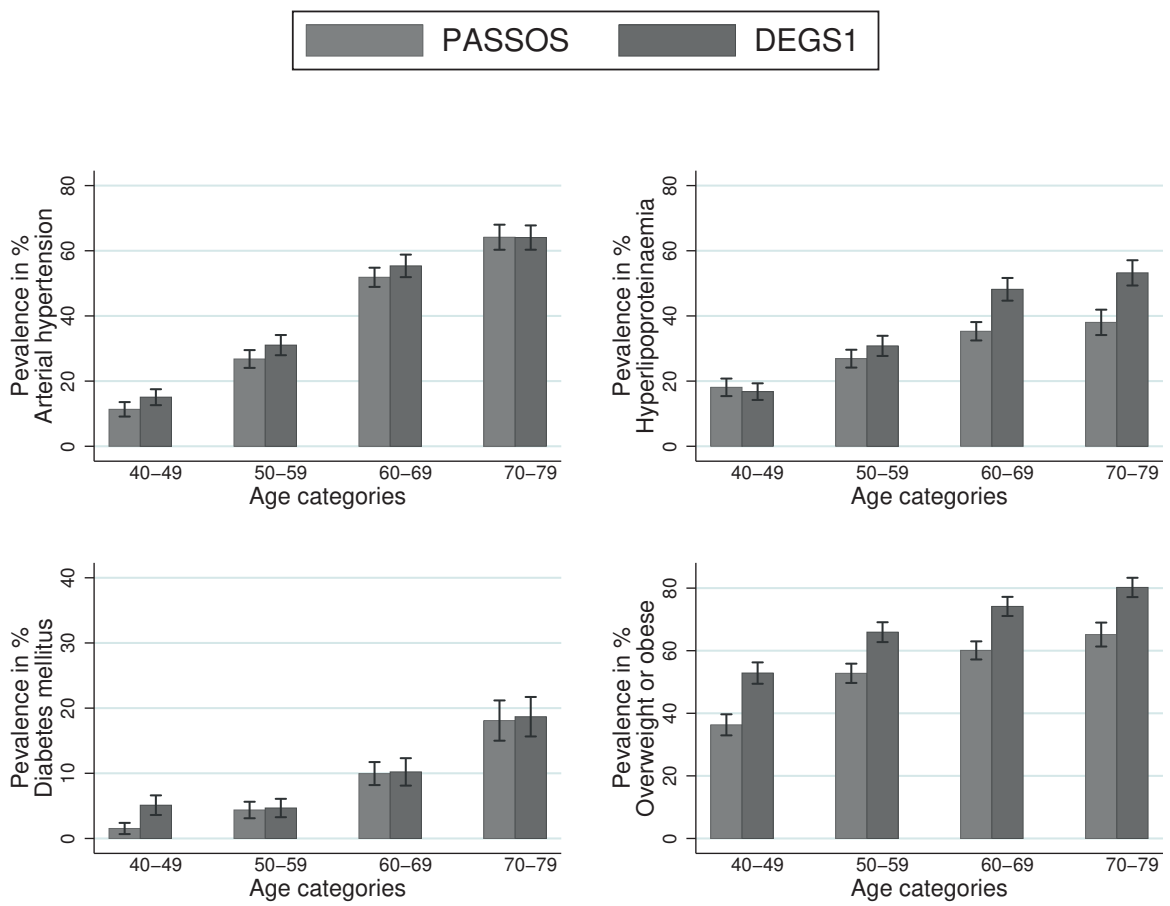

Fig. 2. Prevalence of cardiovascular risk factors at the time of breast cancer diagnosis, stratified by age (PASSOS vs. DEGS1).

vascular risk factors except for myocardial infarction and stroke (not statistically significant).

Comparisons of cancer survivors to similarly aged persons with no cancer history are based on the assumption that there are no physiologic or sociodemographic differences between both groups. However, the incidence of breast cancer is known to be higher among women of higher socioeconomic status compared to the general population, which might be associated with less cardiovascular risk factors and a healthier lifestyle [14].

The data extracted from anesthesia protocols used in the PASSOS heart study is based on self-reported information provided by patients. This information was documented by physicians. As the risk factor assessment is essential for subsequent surgery, we expect an overestimation rather than an underestimation of reported frequencies. However, self-reported information is prone to wellknown limitations, and therefore misclassification of the prior disease status and the presence of risk factors as well as residual confounding through the impact of other factors is likely. The DEGS1 study obtains its information from voluntary self-reporting by 'healthy' subjects in a representative population sample. In particular, self-reports on personal health could lead to a distortion of the actual prevalence; it is possible that interviewed subjects do not recall a disease despite an existing diagnosis. In addition, an existing disease may not (yet) have been discovered. Since prevalences re- 
Table 4. Prevalence of selected cardiovascular risk factors stratified by age; crude and agestandardized prevalence (PASSOS vs. DEGS1).

\begin{tabular}{|c|c|c|c|c|c|c|c|c|c|}
\hline \multirow[t]{2}{*}{ Age, years } & \multirow[t]{2}{*}{ Cardiovascular risk factor } & \multicolumn{4}{|c|}{ PASSOS } & \multicolumn{4}{|c|}{ DEGS1 (CAPI) } \\
\hline & & $\mathrm{N}$ & $\mathrm{n}$ & $\%$ & $95 \%$ CI & $\mathrm{N}$ & $\mathrm{n}$ & $\%$ & $95 \% \mathrm{CI}$ \\
\hline \multirow[t]{5}{*}{$40-49$} & arterial hypertension & 785 & 89 & 11.34 & $9.12 ; 13.56$ & 823 & 124 & $15.07^{\mathrm{d}}$ & $12.62 ; 17.51$ \\
\hline & hyperlipoproteinemia & & 142 & $18.09^{\mathrm{b}}$ & $15.40 ; 20.78$ & & 138 & $16.77^{\mathrm{f}}$ & $14.22 ; 19.32$ \\
\hline & diabetes mellitus & & 12 & 1.53 & $0.67 ; 2.39$ & & 42 & $5.10^{e}$ & $3.6 ; 6.61$ \\
\hline & overweight or obesity & & 285 & 36.31 & $32.94 ; 39.67$ & & 435 & 52.86 & $49.44 ; 56.27$ \\
\hline & any cardiovascular risk factor & & 388 & 49.43 & $45.93 ; 52.92$ & & 523 & 63.55 & $60.26 ; 66.84$ \\
\hline \multirow[t]{5}{*}{$50-59$} & arterial hypertension & 1,008 & 270 & 26.79 & $24.05 ; 29.52$ & 857 & 266 & $31.04^{\mathrm{d}}$ & $27.94 ; 34.14$ \\
\hline & hyperlipoproteinemia & & 271 & $26.88^{\mathrm{b}}$ & $24.15 ; 29.62$ & & 264 & $30.81^{\mathrm{f}}$ & $27.71 ; 33.9$ \\
\hline & diabetes mellitus & & 44 & 4.37 & $3.10 ; 5.63$ & & 40 & $4.67^{\mathrm{e}}$ & $3.26 ; 6.08$ \\
\hline & overweight or obesity & & 532 & 52.78 & $49.70 ; 55.86$ & & 565 & 65.93 & $62.75 ; 69.10$ \\
\hline & any cardiovascular risk factor & & 684 & 67.86 & $64.97 ; 70.74$ & & 692 & 80.75 & $78.11 ; 83.39$ \\
\hline \multirow[t]{5}{*}{$60-69$} & arterial hypertension & 1,105 & 573 & 51.86 & $48.91 ; 54.80$ & 793 & 439 & $55.36^{\mathrm{d}}$ & $51.90 ; 58.82$ \\
\hline & hyperlipoproteinemia & & 390 & $35.29^{b}$ & $32.48 ; 38.11$ & & 382 & $48.17^{f}$ & $44.69 ; 51.65$ \\
\hline & diabetes mellitus & & 110 & 9.95 & $8.19 ; 11.72$ & & 81 & $10.21^{\mathrm{e}}$ & $8.11 ; 12.32$ \\
\hline & overweight or obesity & & 664 & 60.09 & $57.20 ; 62.98$ & & 588 & 74.15 & $71.10 ; 77.20$ \\
\hline & any cardiovascular risk factor & & 896 & 81.09 & $78.78 ; 83.40$ & & 727 & 91.68 & $89.75 ; 93.60$ \\
\hline \multirow[t]{5}{*}{$70-79$} & arterial hypertension & 597 & 383 & 64.15 & $60.31 ; 68.0$ & 637 & 408 & $64.05^{\mathrm{d}}$ & $60.32 ; 67.78$ \\
\hline & hyperlipoproteinemia & & 227 & $38.02^{b}$ & $34.13 ; 41.92$ & & 339 & $53.22^{f}$ & $49.34 ; 57.09$ \\
\hline & diabetes mellitus & & 108 & 18.09 & $15.0 ; 21.18$ & & 119 & $18.68^{\mathrm{e}}$ & $15.65 ; 21.71$ \\
\hline & overweight or obesity & & 389 & 65.16 & $61.34 ; 68.98$ & & 637 & 80.22 & $77.13 ; 83.31$ \\
\hline & any cardiovascular risk factor & & 522 & 87.44 & $84.78 ; 90.10$ & & 603 & 94.66 & $92.92 ; 96.41$ \\
\hline \multirow{5}{*}{$\begin{array}{l}\text { Total, } \\
\text { crude }\end{array}$} & arterial hypertension & 3,496 & 1,316 & 37.64 & $36.04 ; 39.25$ & 3,110 & 1,237 & 39.77 & $38.05 ; 41.50$ \\
\hline & hyperlipoproteinemia & & 1,030 & 29.46 & $27.95 ; 30.97$ & & 1,123 & 36.11 & $34.42 ; 37.80$ \\
\hline & diabetes mellitus & & 274 & 7.84 & $6.95 ; 8.73$ & & 282 & 9.07 & $8.06 ; 10.08$ \\
\hline & overweight or obesity & & 1,871 & 53.52 & $51.86 ; 55.17$ & & 2,099 & 67.49 & $65.85 ; 69.14$ \\
\hline & any cardiovascular risk factor & & 2,491 & 71.25 & $69.75 ; 72.75$ & & 2,545 & 81.83 & $80.48 ; 83.19$ \\
\hline \multirow{5}{*}{$\begin{array}{l}\text { Total, } \\
\quad \text { age- } \\
\text { adjusted }\end{array}$} & arterial hypertension & 3,496 & 1,228 & 35.15 & $33.72 ; 36.58$ & 3,110 & 1,197 & 38.49 & $36.93 ; 40.05$ \\
\hline & hyperlipoproteinemia & & 988 & 28.28 & $26.77 ; 29.78$ & & 1,083 & 34.84 & $33.24 ; 36.43$ \\
\hline & diabetes mellitus & & 264 & 7.55 & $6.68 ; 8.42$ & & 278 & 8.94 & $7.95 ; 9.93$ \\
\hline & overweight or obesity & & 1,809 & 51.77 & $50.10 ; 53.44$ & & 2,081 & 66.92 & $65.29 ; 68.55$ \\
\hline & any cardiovascular risk factor & & 2,412 & 69.00 & $67.48 ; 70.53$ & & 2,517 & 80.92 & $79.58 ; 82.25$ \\
\hline Sum & & $3,496^{\mathrm{a}}$ & & & & 3,110 & & & \\
\hline
\end{tabular}

${ }^{a}$ Missing age information for 1 patient from PASSOS heart study.

${ }^{\mathrm{b}}$ Missing values for hyperlipoproteinemia in PASSOS in 8.79\% (40-49), 9.13\% (50-59), 9.50\% (60-69), $11.72 \%$ (70-79).

${ }^{c}$ Missing values for hyperlipoproteinemia in PASSOS in 7.15\% (40-49), 12.22\% (50-59), 12.9\% (60-69), 16.8\% (70-79).

${ }^{\mathrm{d}}$ Missing values for arterial hypertension in DEGS in 15.92\% (40-49), 12.49\% (50-59), 9.71\% (60-69), and 13.03\% (70-79).

${ }^{\mathrm{e}}$ Missing values for diabetes mellitus in DEGS in 0.36\% (40-49), 1.05\% (50-59), 1.39\% (60-69), 1.73\% (70-79).

${ }^{\mathrm{f}}$ Missing values for hyperlipoproteinemia in DEGS in 1.94\% (40-49), 1.63\% (50-59), 2.27\% (60-69), 4.09\% (70-79).

Numbers in italics: Non-overlapping CIs.

DEGS1 = German Health Interview and Examination Survey for Adults; CAPI = computer-assisted personal interview; $\mathrm{N}=$ population size in age groups; $\mathrm{n}=$ outcome frequency in age groups; $\mathrm{CI}=$ confidence interval. ported in the general population tend to be even higher than those found in breast cancer patients, underreporting of diseases and risk factors in the general population is unlikely.

An additional sensitivity analysis based only on breast cancer patients deceased before 2013 revealed a higher burden of pre-existing cardiac diseases and cardiovascular risk factors compared to the general population.

\section{Comparison with Results from the Literature}

Only few studies dealing with cardiovascular diseases in breast cancer patients already present before breast cancer diagnosis and treatment have been conducted $[7,15,16]$. There are indications of possible adverse effects on postoperative survival. Janssen-Heijnen et al. [16] showed a greater impact of pre-existing comorbidities on overall survival than on postoperative complications. Huang and Camp [15] reported a moderate impact of risk factors such as diabetes mellitus, hypertension, overweight, and smoking on the targeted outcomes such as complications and unplanned additional surgeries. If all risk factors were combined into a categorical exposure variable indicating the number of risk factors present, a sig- 
nificantly increased risk was associated with an increasing number of risk factors. The authors recommended documenting existing cardiovascular diseases in order to better assess risks during surgery and to optimize the treatment of breast cancer patients. Crude prevalences reported for cardiovascular diseases and obesity or overweight in another large population-based cohort study on breast cancer patients in Germany (MARIEplus study) were in line with percentages in the general German population and therefore higher than in the PASSOS study population [7]. The prevalence of hypertension (37.4\%) was lower compared to the general German population as well as compared to the PASSOS study population (45.5 and $48.7 \%$ of women aged $>50$ years).

\section{Strengths and Limitations}

\section{Strengths}

As part of the large-scale multicenter PASSOS heart study, extensive information on cardiovascular health of breast cancer patients was systematically recorded. The detailed data allow a comparison of the frequencies of cardiac diseases and risk factors between breast cancer patients and the general female German population. Such a comparison has not yet been performed. DEGS1 is a nationwide, population-representative study that allows for generalizations of the reported prevalences for the adult population in Germany.

\section{Limitations}

The PASSOS heart study considers anamnesis data collected between 1998 and 2008. The first wave of the DEGS1 survey was conducted between 2008 and 2011. Gößwald et al. [13] showed that prevalences reported in 1998 (BGS98) only slightly differed from those reported in DEGS1. In this respect, we do not expect any distortion of results due to the choice of a more recent comparison population.

Stang et al. [17] reviewed age-standardized prevalences of arterial hypertension, diabetes mellitus, and obesity across German federal states in the context of a selective literature search and screening of official statistics. In this comparison, prevalences of arterial hypertension and diabetes mellitus were in the median range in Baden-Württemberg and Rhineland-Palatinate, as is also shown in our comparisons.

Being overweight is a recognized risk factor for postmenopausal receptor-positive breast cancer [18]. Our data show a lower prevalence of being overweight or obese in breast cancer patients compared to the general female population. In the study by Stang et al. [17], the second lowest prevalence of obesity was observed in Baden-Württemberg, which may explain the difference observed in our study: study participants from the PASSOS heart study were recruited in Mainz and Ulm. The different prevalence may furthermore be caused by differences between cities and rural areas.

Smoking habits could not be considered in our analysis since smoking information was not documented at the time of diagnosis. Data from subsequent follow-up examinations are less suitable for our analysis due to the time-delayed recording. The prevalence of smoking at the time of diagnosis would likely be more conservative if we used follow-up data, since patients may change their habits following a breast cancer diagnosis.

Finally, our study results might be influenced by only considering patients who received breast cancer surgery. These women might have a different cardiovascular risk profile compared to patients who were not recommended for surgery possibly due to a worse cardiac health status. However, this possible bias is negligible since this medical situation applies to only rare cases (personal communication).

\section{Conclusion}

The improved prognosis in breast cancer patients increases the relevance of investigating potential comorbidities and cardiovascular risk factors. Breast cancer therapy is associated with an increased risk of cardiovascular late effects [6]. It is therefore important to consider any prior cardiac diseases for prevention purposes. Pre-existing cardiac diseases and cardiovascular risk factors are common in both study populations, being slightly less frequent in the PASSOS cohort than in the general female population in Germany. Cardiovascular diseases, prevalent before the oncological therapy, should be carefully monitored and taken into account when making therapy decisions, and interventions should be adapted accordingly on an individual basis.

\section{Ethical Approval and Consent to Participate}

The PASSOS study was approved by the Ethics Committee of RhinelandPalatinate, Mainz and the Ethics Committee of the University of Ulm.

\section{Funding}

This study was funded by the Federal Ministry of Education and Research (BMBF) (02NUK026B). The study sponsor had no influence on patient identification, the survey, analysis and interpretation of results, writing of the manuscript, or the decision to submit the manuscript for publication.

\section{Acknowledgment}

We are grateful to all participants of the PASSOS heart study. In addition, we thank Dagmar Lautz und Dorothea Niehoff for their valuable technical assistance. Kathy Taylor kindly proofread the manuscript and checked the wording and English grammar.

\section{Disclosure Statement}

The authors declare no conflict of interest. 


\section{References}

RKI: Krebs in Deutschland 2011/2012. Robert KochInstitut und die Gesellschaft der epidemiologischen Krebsregister in Deutschland e.V., 2015.

- Van Ewijk RJ, Schwentner L, Wockel A, Konig J, Kreienberg R, Blettner M: Trends in patient characteristics, treatment and survival in breast cancer in a nonselected retrospective clinical cohort study of 2,600 patients. Arch Gynecol Obstet 2013;287:103-110.

3 Patnaik JL, Byers T, Diguiseppi C, Denberg TD, Dabelea $\mathrm{D}$ : The influence of comorbidities on overall survival among older women diagnosed with breast cancer. J Natl Cancer Inst 2011;103:1101-1111.

4 Darby SC, Ewertz M, McGale P, Bennet AM, BlomGoldman U, Bronnum D, Correa C, Cutter D, Gagliardi G, Gigante B, Jensen MB, Nisbet A, Peto R, Rahimi K, Taylor C, Hall P: Risk of ischemic heart disease in women after radiotherapy for breast cancer. N Engl J Med 2013;368:987-998.

5 Schlitt A, Jordan K, Vordermark D, Schwamborn J, Langer T, Thomssen C: Kardiotoxizität onkologischer Therapien. Dtsch Arztebl Int 2014;111:161-168.

6 Schmitz KH, Prosnitz RG, Schwartz AL, Carver JR: Prospective surveillance and management of cardiac toxicity and health in breast cancer survivors. Cancer 2012;118:2270-2276.

7 Obi N, Gornyk D, Heinz J, Vrieling A, Seibold P, Chang-Claude J, Flesch-Janys D: Determinants of newly diagnosed comorbidities among breast cancer survivors. J Cancer Surviv 2014;8:384-393.
Merzenich $\mathrm{H}$, Bartkowiak D, Schmidberger $\mathrm{H}$, Schmidt M, Schwentner L, Wiegel T, Woeckel A, Wollschlager D, Blettner M: 3D conformal radiotherapy is not associated with the long-term cardiac mortality in breast cancer patients: a retrospective cohort study in Germany (PASSOS-Heart Study). Breast Cancer Res Treat 2017;161:143-152.

9 Wollschlager D, Merzenich H, Schwentner L, Janni W, Wiegel T, Bartkowiak D, Wockel A, Schmidt M, Schmidberger $\mathrm{H}$, Blettner M: Self-reported long-term cardiac morbidity in breast cancer patients: a retrospective cohort study in Germany (PASSOS Heart Study). Breast Cancer Res Treat 2017;163:595-604.

10 Kurzeder C, König J, Wischnewsky M, Koretz K, Moeller P, Wiegel T, Brambs HJ, Blettner M, Kreienberg R: Impact of guideline conform local-regional treatment on survival of breast cancer patients: an analyses of the BRENDA-study group. J Clin Oncol 2008;26:abstr 612.

11 Schwentner L, Wockel A, Konig J, Janni W, Ebner F, Blettner M, Kreienberg R, van Ewijk R: Adherence to treatment guidelines and survival in triple-negative breast cancer: a retrospective multi-center cohort study with 9,156 patients. BMC Cancer 2013;13:487.

12 Kamtsiuris P, Lange M, Hoffmann R, Schaffrath Rosario A, Dahm S, Kuhnert R, Kurth BM: The first wave of the German Health Interview and Examination Survey for Adults (DEGS1): sample design, response, weighting and representativeness (Article in German). Bundesgesundheitsblatt Gesundheitsforschung Gesundheitsschutz 2013;56:620-630
Gosswald A, Schienkiewitz A, Nowossadeck E, Busch MA: Prevalence of myocardial infarction and coronary heart disease in adults aged 40-79 years in Germany: results of the German Health Interview and Examination Survey for Adults (DEGS1) (Article in German). Bundesgesundheitsblatt Gesundheitsforschung Gesundheitsschutz 2013;56:650-655.

14 Lundqvist A, Andersson E, Ahlberg I, Nilbert M, Gerdtham U: Socioeconomic inequalities in breast cancer incidence and mortality in Europe - a systematic review and meta-analysis. Eur J Public Health 2016;26:804-813.

15 Huang BZ, Camp MS: Burden of preoperative cardiovascular disease risk factors on breast cancer surgery outcomes. J Surg Oncol 2016;114:144-149.

16 Janssen-Heijnen MLG, Maas HAAM, Houterman S, Lemmens VEPP, Rutten HJT, Coebergh JWW: Comorbidity in older surgical cancer patients: influence on patient care and outcome. Eur J Cancer 2007;43: 2179-2193.

17 Stang A, Stang M: Kardiovaskuläre Risikofaktoren im Bundeslandvergleich. Dtsch Arztebl Int 2014;111:530536.

18 Suzuki R, Orsini N, Saji S, Key TJ, Wolk A: Body weight and incidence of breast cancer defined by estrogen and progesterone receptor status - a meta-analysis. Int J Cancer 2009;124:698-712. 